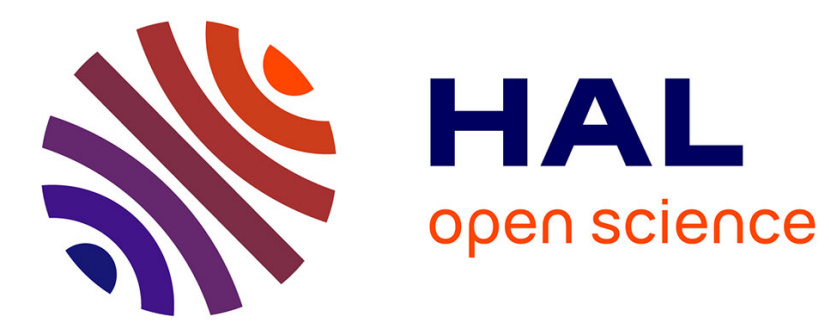

\title{
Nucleosomes represent a physical barrier for cleavage activity of DNA topoisomerase $I$ in vivo
}

Francesca Di Felice, Francesco Chiani, Giorgio Camilloni

\section{To cite this version:}

Francesca Di Felice, Francesco Chiani, Giorgio Camilloni. Nucleosomes represent a physical barrier for cleavage activity of DNA topoisomerase I in vivo. Biochemical Journal, 2007, 409 (3), pp.651-656. 10.1042/BJ20070893 . hal-00478842

\section{HAL Id: hal-00478842 \\ https://hal.science/hal-00478842}

Submitted on 30 Apr 2010

HAL is a multi-disciplinary open access archive for the deposit and dissemination of scientific research documents, whether they are published or not. The documents may come from teaching and research institutions in France or abroad, or from public or private research centers.
L'archive ouverte pluridisciplinaire HAL, est destinée au dépôt et à la diffusion de documents scientifiques de niveau recherche, publiés ou non, émanant des établissements d'enseignement et de recherche français ou étrangers, des laboratoires publics ou privés. 


\section{Nucleosomes represent a physical barrier for cleavage activity of DNA topoisomerase I in} vivo.

Francesca Di Felice ${ }^{*}$, Francesco Chiani* and Giorgio Camilloni*,\$.

* Dipartimento di Genetica e Biologia Molecolare Università di Roma La Sapienza

Roma, 00185 Italy

${ }^{\S}$ Istituto Biologia e Patologia Molecolari-CNR- Roma, 00185, Italy

Corresponding author:

Giorgio Camilloni

giorgio.camilloni@uniroma1.it;

Università di Roma La Sapienza, Dipartimento di Genetica e Biologia Molecolare, P.le. A. Moro 5, 00185 Roma, Italy.

Tel: +390649912808;

Fax: +390649912500

Running title: DNA topoisomerase I and nucleosomes 


\section{SYNOPSIS}

DNA topoisomerase I together with the other cellular DNA topoisomerases releases the torsional stress from DNA caused by processes such as replication, transcription and recombination. Despite the well defined knowledge of its mechanism of action, DNA topoisomerase I in vivo activity has been only partially characterized. In fact the basic question concerning the capability of the enzyme to cleave and rejoin DNA wrapped around histone octamer remains still unanswered.

By studying both in vivo and in vitro the cleavage activity of DNA topoisomerase I in the presence of camptothecin on a repeated trinucleotide sequence, (TTA) 35 , lying in the chromosome XIII of Saccharomyces cerevisiae, we can conclude that nucleosomes represent a physical barrier for the enzyme activity.

Keywords: chromatin; DNA structure; DNA topoisomerase I; nucleosomes; S. cerevisiae; trinucleotide repeats.

\section{INTRODUCTION}

DNA topology in eukaryotic nuclei is controlled by a specific class of proteins: DNA topoisomerases [1]. These enzymes are classified into two groups depending on their catalytic activity: type I enzymes change DNA topology by breaking and rejoining one DNA strand at a time, while type II act contemporaneously on both strands. In eukaryotes, DNA topoisomerase IB reduces the torsional stress arising from basic DNA transactions like transcription, replication and recombination [2].

Although DNA topoisomerase I is involved in such important functions, the enzyme is not essential in S. cerevisiae [3].

Despite the well-known mechanisms of DNA topoisomerase I activity and the susceptibility to different inhibitors [4], its accessibility to the DNA in vivo has been poorly investigated. To date, evidences about the in vivo DNA topoisomerase I cleavage activity only refer to nucleosome free regions [5-7] and it is not known whether nucleosomes could represent a barrier for DNA topoisomerase I to access DNA. In fact it has been largely demonstrated that nucleosomal complexes interfere with the accessibility of transcription and replication factors on DNA [8-9]. 
Recently, Roca and coworkers demonstrated that DNA topoisomerase II relaxes nucleosomal DNA much faster than DNA topoisomerase I by analyzing the relaxing activities on different nucleosomal substrates suggesting that, in vivo, DNA topoisomerase I more than DNA topoisomerase II, could be impeded by nucleosomal structures in cleaving and rejoining DNA [10]. Actually the DNA topoisomerase I cleavage reaction on in vivo sequences has not been yet studied on substrates containing or not nucleosomes. In order to answer this question we evaluated the DNA topoisomerase I cleavage activity on the natural (TTA) ${ }_{35}$ repeated sequence, both in vitro and in vivo. This sequence contains structural features presumably favouring both the DNA topoisomerase I site-specific cleavage activity and nucleosome assembly. In fact, the TTA sequence has been shown to be intrinsically flexible [11] and the TA step is locally bent [12]. These two features (i.e. flexibility and local bending) and the previous observation that DNA topoisomerase I in vitro cleaves efficiently the bent TA step [13] rendered the (TTA) 35 repeat a good substrate by which to evaluate the capability of DNA topoisomerase I to cleave a specific sequence, possibly in the presence of nucleosomes. In addition, using a strain [14] where the H4 synthesis is under the GAL1 promoter, we were able to study the DNA topoisomerase I activity on a sequence where the nucleosome organization is lost or maintained in glucose or galactose respectively. This approach allowed us to study, in vivo, the DNA topoisomerase I reaction on different chromatin arrangements.

\section{EXPERIMENTAL}

\section{Strains, plasmids and culture media}

Strains: W303-1a (Mata, ade 2-1, ura 3-1, his 3-11,15, trp1-1, leu 2-3,112, can1-100); AMR51 (Mata, ade 2-1, ura 3-1, his 3-11,15, trp1-1, leu 2-3,112, can1-100, TOP1::leu2) kindly provided by R. Sternglanz; UKY403 (Mata, ade 2-101, his -4200, leu 2-3,112, lys2-801, ura3-52, trp1-

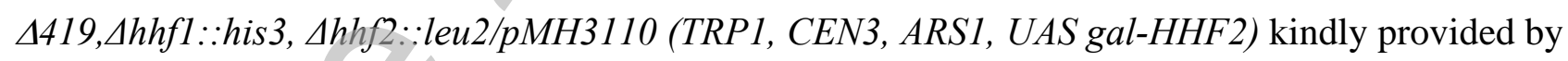
M.Grunstein.

Plasmid pFDFG1a: The sequence -422 to +612 (from PRE8 ATG) encompassing the (TTA) 35 region, was PCR amplified and cloned in the EcoRI site of the yeast vector PADH426 [15] yielding the plasmid pFDFG1a.

Culture media: cells were grown in minimal YNB [16], supplemented with $2 \%$ glucose or $2 \%$ galactose when appropriate. 


\section{Enzymes and chemicals.}

Restriction enzymes, T4 polynucleotide kinase and Micrococcal nuclease were from Roche. Vent(exo־) polymerase from New England Biolabs; Zymolyase 100T from Seikagaku, Tokyo, Japan; CPT from Sigma; radiochemicals from Amersham.

\section{Chromatin analysis}

Micrococcal Nuclease treatment was performed as previously described [17]. MNase (2 and 4 units) was added to different aliquots of spheroplasts preparation and the samples were incubated at $25^{\circ} \mathrm{C}$ for 20 minutes. The DNA was then deproteinized and ethanol precipitated. RNase treatment was also performed.

\section{Low resolution analysis}

Indirect end labeling analysis [18] was performed as follows: after treatment with the appropriate restriction enzymes, the samples were electrophoresized in $1.2 \%$ agarose gels $(1.75 \mathrm{volts} / \mathrm{cm})$, transferred onto nitrocellulose membrane, hybridized to the TTA probe and detected by autoradiography.

\section{In vivo induction of CPT dependent DNA topoisomerase I cleavage sites}

Cells were treated for cleavage sites induction as previously [7]. Three different aliquots were incubated with 50-100-200 $\mu \mathrm{M}$ CPT for 2 minutes at R.T. The reaction was stopped with 1\% SDS, 5 mM EDTA. The DNA was then deproteinized and ethanol precipitated. RNase treatment was also performed.

\section{Oligonucleotide primers and probes}

Oligonucleotides sequences used in the extension reactions:

VEC: 5'-GGTATCGATAAGCTTGATATCG-3'

r3: 5'-CGCGTTTCCGTATTTTCCGC-3'

The oligonucleotide VEC lies at position -449 from PRE8 ATG of the cloned sequence containing the (TTA) $)_{35}$ in pFDFG1a. The r3 oligonucleotide used lies at position -267/-248 bp from the 35S 35S RNA RIS. 5'-end labelling using $\left(\gamma-{ }^{32} \mathrm{P}\right) \mathrm{ATP}$ and T4 polynucleotide- kinase was performed according to [19].

The probe TTA, annealing at positions $+436 /+232$ bp, from the ATG of PRE8 gene, was labelled by random priming.

Multiple-round primer extension and high resolution analysis of in vivo MNase and DNA topoisomerase I cleavage sites.

$1 / 2 \mu \mathrm{g}$ of genomic DNA were reacted with Vent polymerase and end-labelled oligonucleotide as described [20]. The samples were cycled 25 times through the following steps: $95^{\circ} \mathrm{C}$ for 5 min., $58^{\circ} \mathrm{C}$ for $10 \mathrm{~min}$., and $76^{\circ} \mathrm{C}$ for $3 \mathrm{~min}$. (conditions for oligonucleotide VEC). The extension 
products were phenol extracted, ethanol precipitated, dissolved in formamide buffer and analysed by $6 \%$ denaturing polyacrylamide gel electrophoresis.

\section{In vitro DNA topoisomerase I cleavage sites.}

The reactions were performed as described [21] reacting 1, 5 and 10 Units of purified DNA topoisomerase I (calf thymus) with 1-2 ng of end-labeled DNA. The reactions were carried out in $10 \mathrm{mM}$ Tris-HCl, pH8.0, $10 \mathrm{mMNaCl}, 1 \mathrm{mM} \mathrm{MgCl} 2$ and $100 \mu \mathrm{M}$ CPT for $15 \mathrm{~min}$ at $37^{\circ} \mathrm{C}$. The DNA substrate for cleavage reaction was EcoRI excised from pFDFG1a. After restriction with XhoI/XbaI the fragment was 3'-labeled, purified and further digested with SmaI or HindIII in order to uniquely orient the labelling.

\section{RESULTS}

We selected the trinucleotide repeat (TTA) 35 lying in the intergenic region of the chromosome XIII of $S$. cerevisiae, between the RPM2 and PRE8 genes. This sequence, as reported above, contains the structural elements presumably suitable to be susceptible to DNA topoisomerase I site-specific activity and to assemble nucleosomes.

\section{In vitro high resolution analysis of DNA topoisomerase I cleavage sites on (TTA) ${ }_{35}$ repeat.}

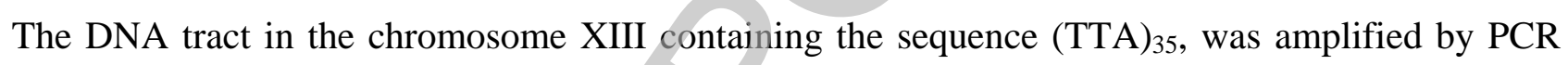
with two oligonucleotides annealing at positions -474 and +755 relative to the PRE 8 ATG. The resulting fragment was cloned into the yeast vector pADH426, yielding the pFDFG1a plasmid. The XhoI/XbaI fragment from pFDFG1a was 3'-labeled, purified and further digested with SmaI in order to uniquely orient the labelling and to study the DNA topoisomerase I cleavages with a nucleotide level of resolution. The fragment was reacted in vitro with increasing amounts of calf thymus DNA topoisomerase I (triangle in Figure 1) in the presence of Camptothecin (CPT); this drug inhibits the rejoining reaction of DNA topoisomerase I thus creating, in the presence of a denaturing agent, single strand breaks and behaving like a single strand nuclease. As reported in Figure 1, DNA topoisomerase I strongly recognizes the TTA repeats (arrows). The regularly repeated sequence is highly cleaved (from -312 to -216) and TTA repetitions in the flanking regions are also efficiently cleaved. Asterisks indicate cleavage sites not occurring on a TTA sequence.

\section{In vivo analysis of DNA topoisomerase I cleavage sites on the (TTA) ${ }_{35}$ repeat.}

Given the results reported in Figure 1 we evaluated the DNA topoisomerase I cleavage activity on the same DNA sequence in vivo. WT and AMR51 (Ltopl) cells, transformed with pFDFG1a, were analyzed for cleavage sites induction as previously reported [7, 20]. Briefly: spheroplasts from WT 
and $\Delta$ top 1 cells were permeabilized with nystatin [17] and treated with different amounts of CPT (triangles in Figure 2). DNA was then purified and primer extended from the labelled oligonucleotide VEC (annealing on the vector sequence at -449 bp from PRE 8 ATG contained in the fragment cloned in pADH426). Only a weak cleavage profile is observed in the WT strain (Figure 2). The only detectable sites localize at the flanking regions of the triplet (arrows). In the Atopl strain no cleavage sites are observed. Taken together, these data suggest that DNA topoisomerase I poorly reacts in vivo with the (TTA) $)_{35}$ repeat despite its in vitro high reactivity towards the same sequence (Figure 1).

\section{(TTA) $)_{35}$ repeat is engaged in a positioned nucleosome in vivo.}

Results reported in Fig 2 indicate that DNA topoisomerase I does not efficiently react with the $(\mathrm{TTA})_{35}$ tract in vivo. Could this low cleavage activity on the repeated sequence be due to its chromatin organization? In order to clarify this point we studied the nucleosome positioning on this DNA sequence by in vivo analysis with Micrococcal Nuclease (MNase).

Spheroplasts from WT cells, transformed with pFDFG1a, were treated with nystatin, digested with different amounts of MNase (triangles in Figure 3) and DNA was purified. Results are shown in Figure 3 Panel A: the in vivo treated samples (chromatin) were compared with those digested in vitro (naked DNA) to provide the susceptibility of deproteinized DNA to MNase. This comparison shows protected regions representing nucleosomal particles based on their dimension (140-160 bp) (ellipses in the figure). Nucleosome reported as \#1 occupies positions from -160 bp to - 330bp in the chromatin samples, while in the naked DNA the same region appears strongly digested. This region encompasses the (TTA) 35 region $(-214$ to -318$)$.

To increase the mapping resolution, we primer-extended the DNA of different aliquots from the same samples with the labeled oligonucleotide VEC. The high resolution data reported in Panel B show that the $(\mathrm{TTA})_{35}$ repeat is almost completely protected by the nucleosome \#1 (from -349/324bp to -189bp on multiple positions, filled arrows in Figure3 Panel B) as shown by comparing the chromatin samples with the naked DNA. In addition MNase hypersensitive sites (empty arrows) localize at the flanking regions of the mapped nucleosome.

Taken together the data reported in Figure 3 demonstrate that the (TTA) 35 repeat is actually organized in a family of positioned nucleosomes covering multiple positions, all sharing the TTA repeat in the middle of the particle.

\section{In vivo chromatin organization of the (TTA) ${ }_{35}$ sequence after nucleosome removal.}


Could the positioned nucleosome on (TTA) $)_{35}$ be responsible for DNA topoisomerase I failure in generating efficient cleavage sites in vivo? In order to verify this hypothesis we analyzed the in vivo DNA topoisomerase I cleavage activity in a yeast strain lacking both $\mathrm{H} 4$ genes and carrying one copy of H4 histone under the GAL1 promoter [14]. This implies that in the presence of glucose as carbon source, the chromatin organization will be lost and DNA should become accessible in vivo both to MNase and DNA topoisomerase I.

Yeast UKY403 cells, transformed with pFDFG1a, grown in galactose, were splitted in two aliquots, washed and resuspended in a growth medium containing galactose or glucose. After 4 hours of growth, spheroplasts were treated with different amounts of MNase. The digestion profiles shown in Figure 4, Panel A, (bulk DNA), indicate that in the GAL grown cells the nucleosomal ladder is obtained, while in the GLU cultures, a smear appears in the corresponding region of the gel. This suggests that MNase freely accesses DNA when the nucleosomal barriers are absent.

When the samples from GAL or GLU cultures were analyzed by end-labeling technique [18] as in Figure3 Panel A, a specific difference is observed (Figure 4. Panel B): GAL samples show protection of the TTA region towards MNase (nucleosome \#1), as reported in Figure 3; conversely, GLU samples show a diffuse accessibility to MNase on the (TTA) 35 sequence similar to that of the naked DNA.

\section{DNA topoisomerase $I$ in vivo cleaves the (TTA) ${ }_{35}$ sequence after nucleosomes removal.}

The data reported in Figure 4, Panel B, demonstrate that the chromatin organization of the (TTA) 35 repeat is completely lost when cells were grown in glucose. Since in these conditions MNase accesses (TTA) $)_{35}$ repeat with the same efficiency in vivo end in vitro, we wanted to evaluate whether also DNA topoisomerase I could reach this sequence when chromatin is not organized any more. We then analyzed the same cells for DNA topoisomerase I cleavage sites induction at high resolution (Figure 5 Panel, A). The samples were primer extended starting from the labeled oligonucleotide VEC: the reactivity of DNA topoisomerase I in the GLU culture is highly enhanced compared to that observed in the GAL culture. In addition, the cleavage profile of GLU samples (arrows) almost completely overlaps to that obtained in vitro (see Figure 1). Then we analyzed the same samples in a different chromosomal location (the NTS2 region of the rDNA) as a control. In fact, we have previously shown [7, 20] that in this nucleosome free region [22] a strong DNA topoisomerase I cleavage site occurs at -171 bp from R.I.S [7]. Results are shown in Figure 5, Panel B. When the and the oligonucleotide r3 [-268bp from the 35S RNA start site (R.I.S.)] was primer extended both conditions (GLU/GAL, i.e. nucleosomes presence/absence respectively) show the 171 cleavage site, demonstrating the same reactivity of DNA topoisomerase I in a nucleosome free 
region when $\mathrm{H} 4$ transcription is on or off (Figure 5, Panel B). Taken together the data reported in Figure 5, Panels A and B, demonstrate that nucleosomes represent a barrier for localized DNA topoisomerase I activity.

In order to exclude that the observed differences in accessibility to TTA region are due to a different amounts of DNA topoisomerase I in the two growth conditions, we measured both TOP1 mRNA production and DNA relaxing activity (not shown). All evaluations showed a slight increase in mRNA and DNA topoisomerase I activity in cells from GLU cultures; however such a small difference does not justify the strong enhancements observed in the in vivo cleavage profile (Figure 5 PanelA).

\section{DISCUSSION}

It is generally accepted that all sequences in DNA can be substrates for DNA topoisomerase I relaxing activity, even though DNA topoisomerase I seems to have a higher reactivity towards some particular sequences showing structural features like DNA bending [13, 23]. The high resolution mapping of in vivo DNA topoisomerase I site specific activity concerns nucleosome free regions [57]. In order to verify whether nucleosomes affect DNA topoisomerase I site-specific cleavages, we studied a natural DNA sequence repeating 35 times the TTA trinucleotide. Such a sequence provides: the locally bent TA step [12], presumably a good substrate for local DNA topoisomerase I cleavage induction and an intrinsic flexibility [11] potentially useful to efficiently assemble nucleosome.

The TTA trinucleotide repeat has proved to be efficiently cleaved in vitro by DNA topoisomerase I (Figure 1) in the repeated tract and also in its surrounding regions, confirming the preference of the enzyme for this sequence. Conversely, an in vivo approach showed a reduced reactivity of the same sequence towards DNA topoisomerase I. Thus, it is conceivable to hypothesize that chromatin structure affects DNA topoisomerase I cleavages. Recently Roca and coworkers [10] observed that DNA topoisomerase II is more efficient than DNA topoisomerase I in releasing topological stress from nucleosomal substrates; this supports the hypothesis that nucleosomes may represent a barrier for DNA topoisomerase I activity. At this purpose a distinction between the global relaxing activity and the local site specific cleavage reaction, should be taken under consideration. In fact a different chromatin organization in a given substrate, could be not determinant in the whole relaxing activity of DNA topoisomerase I: indeed the enzyme can release torsional stress acting on different sites in the substrate. Conversely, when a given sequence is analyzed in terms of cleavage activity exerted by DNA topoisomerase I, the absence/presence of a nucleosome could be very relevant and this latter point was investigated by our experimental system. 
In order to verify this hypothesis, we studied the in vivo chromatin organization of the (TTA) $)_{35}$ tract. As reported in Figure 3, a positioned nucleosome occupies the (TTA) 35 region possibly hindering the DNA topoisomerase I cleavage activity. Then we employed a yeast strain carrying a plasmid in which the H4 gene is under the GAL1 promoter. This allowed us to switch on/off the synthesis of this histone, depending on the carbon source. In the GAL condition the presence of nucleosomes impairs DNA topoisomerase I approach to the (TTA) ${ }_{35}$ sequence. Conversely, in the GLU condition, when H4 production is repressed, no organized chromatin is observed on the TTA repeat and DNA topoisomerase I increases its sequence specific activity, particularly on the (TTA) 35 tract (Figure 4).

In order to evaluate the cleavage difference of DNA topoisomerase I in vivo, in vitro and when chromatin is destructured (H4 no more synthesized in Glucose medium), we quantified the DNA topoisomerase I digestion profiles and the data are compared in Figure 6. As it appears, when chromatin is regularly organized (in vivo and in vivo GAL, see also chromatin profiles in Figs.3 and 4) about $5-6 \%$ of the digested material is represented by the TTA repeat. Conversely the relative digestion of the TTA repeat when samples are reacted in vitro with DNA topoisomerase I reaches values near to $30 \%$. Also the dissolution of regularly organized chromatin allows DNA topoisomerase I to digest the TTA repeat in vivo, but with an efficiency of 15\% (about three times higher than on the regular chromatin).

Thus we can conclude that: i) DNA topoisomerase I efficiently reacts with the TTA repeat; ii) the (TTA) ${ }_{35}$ sequence, the longest and most stable among the simple repeated sequences in $S$. cerevisiae [24], is organized in a positioned nucleosome and possibly this can account for its high stability: in fact each nucleosome stores one negative supercoil [25-26], thus preventing DNA denaturation and induction of conformational alterations responsible for genetic instability [27]; iii) the positioned nucleosome on the (TTA) 35 sequence represents a hindrance to the DNA topoisomerase I activity. This last conclusion, based on the GLU/GAL experiments, represents the first formal evidence that DNA topoisomerase I cannot react with nucleosomal DNA.

Despite the well known chemistry concerning the DNA topoisomerase I mechanism of action and the interference with small inhibitors largely employed in chemotherapy, so far no details are known about the possible interference that nucleosome could represent in vivo towards topoisomerization reaction. Here we provide direct evidences clarifying this task. 


\section{ACKNOWLEDGEMENTS}

This work was partially supported by the Istituto Pasteur-Fondazione Cenci Bolognetti Universita' di Roma "La Sapienza" and by "Programmi di Ricerca Scientifica di Rilevante Interesse Nazionale" MIUR 2006. We are thankful to M. Grunstein for UKY403 and to R. Sternglanz for AMR51 (top 14). We also acknowledge M.Caserta for critical reading of the manuscript. 


\section{REFERENCES}

1 Wang, J.C. (2002) Cellular roles of DNA topoisomerases: a molecular perspective. Nat. Rev. Mol. Cell. Biol. 3, 430-440.

2 Champoux, J.J. (2001) DNA topoisomerases: structure, function, and mechanism. Annu. Rev. Biochem. 70, 369-413.

3 Thrash, C., Voelkel, K., Di Nardo, S. and Sternglanz, R. (1984) Identification of Saccharomyces cerevisiae mutants deficient in DNA topoisomerase I activity. J. Bioll. Chem. 259, 1375-1377.

4 Pommier, Y. (2006) Topoisomerase I inhibitors: camptothecins and beyond. Nat. Rev. Cancer 6, 789-802.

5 Bonven, B.J., Gocke, E. and Westergaard, O. (1985) A high affinity topoisomerase I binding sequence is clustered at DNAase I hypersensitive sites in Tetrahymena R-chromatin. Cell 41, 541551.

6 Stewart, A.F., Herrera, R.E. and Nordheim, A. (1990) Rapid induction of c-fos transcription reveals quantitative linkage of RNA polymerase II and DNA topoisomerase I enzyme activities. Cell 60, 141-149.

7 Vogelauer, M. and Camilloni, G. (1999) Site-specific in vivo cleavages by DNA topoisomerase I in the regulatory regions of the $35 \mathrm{~S}$ rRNA in Saccharomyces cerevisiae are transcription independent. J. Mol. Biol. 293, 19-28.

8 Falbo, K.B. and Shen, X. (2006) Chromatin remodeling in DNA replication. J. Cell. Biochem. 97, 684-689.

9 Khorasanizadeh, S. (2004) The nucleosome: from genomic organization to genomic regulation. Cell 116, 259-272.

10 Salceda, J., Fernandez, X. and Roca, J. (2006) Topoisomerase II, not topoisomerase I, is the proficient relaxase of nucleosomal DNA.. EMBO J 25, 2575-2583.

11 Travers, A.A. (2004) The structural basis of DNA flexibility. Philos Transact A Math Phys Eng Sci. 362, 1423-1438.

12 Ulyanov, N.B., Bauer, W.R. and James, T.L. (2002) High-resolution NMR structure of an ATrich DNA sequence. J. Biomol. NMR 22, 265-280.

13 Camilloni, G., Caserta, M., Amadei, A. and Di Mauro, E. (1991) The conformation of constitutive DNA interaction sites for eukaryotic DNA topoisomerase I on intrinsically curved DNAs. Biochim. Biophys. Acta 1129, 73-82.

14 Kim, U.J., Han, M., Kayne, P. and Grunstein, M. (1988) Effects of histone H4 depletion on the cell cycle and transcription of Saccharomyces cerevisiae. EMBO J. 7, 2211-2219. 
15 Mumberg, D., Muller, R. and Funk, M. (1995) Yeast vectors for the controlled expression of heterologous proteins in different genetic backgrounds. Gene 156, 119-122.

16 Sherman, F., Fink, G.R. and Lawrence, C. (1983) Methods in Yeast Genetics, Cold Spring Harbor University Press, Cold Spring Harbor.

17 Venditti, S. and Camilloni, G. (1994) In vivo analysis of chromatin following nystatin-mediated import of active enzymes into Saccharomyces cerevisiae. Mol. Gen. Genet. 242, 100-104.

$18 \mathrm{Wu}, \mathrm{C}$. (1980) The 5' ends of Drosophila heat shock genes in chromatin are hypersensitive to DNase I. Nature 286, 854-860.

19 Sambrook, J, Fritsch, E.F. and Maniatis, T (1989) Molecular Cloning, 2nd Ed., Cold Spring Harbor University Press, Cold Spring Harbor.

20 Di Felice, F., Cioci, F. and Camilloni G. (2005) FOB1 affects DNA topoisomerase I in vivo cleavages in the enhancer region of the Saccharomyces cerevisiae ribosomal DNA locus. Nucleic Acids Res. 33, 6327-6337.

21 Camilloni, G., Di Martino, E., Di Mauro, E. and Caserta, M. (1989) Regulation of the function of eukaryotic DNA topoisomerase I: topological conditions for inactivity. Proc. Natl. Acad. Sci. U.S.A. 86, 3080-3084.

22 Vogelauer M., Cioci F. and Camilloni G. (1998) DNA protein-interactions at the Saccharomyces cerevisiae 35 S rRNA promoter and in its surrounding region. J. Mol. Biol. 275, 197-209.

23 Krogh, S., Mortensen, U.H., Westergaard, O. and Bonven, B.J. (1991) Eukaryotic topoisomerase I-DNA interaction is stabilized by helix curvature. Nucleic Acids Res. 19, 12351341.

24 Young, E.T., Sloan, J.S. and Van Riper, K. (2000) Trinucleotide repeats are clustered in regulatory genes in Saccharomyces cerevisiae. Genetics 154, 1053-1068.

25 Finch, J.T., Lutter, L.C., Rhodes, D., Brown, R.S., Rushton, B., Levitt, M. and Klug, A. (1977) Structure of nucleosome core particles of chromatin. Nature 269, 29-36.

26 Pearson, C.E. and Sinden, R.R. (1998) Trinucleotide repeat DNA structures: dynamic mutations from dynamic DNA. Curr. Opin. Struct .Biol. 8, 321-330.

27 Ohshima, K., Kang, S., Larson, J.E. and Wells, R.D. (1996) TTA.TAA triplet repeats in plasmids form a non-H bonded structure. J. Biol. Chem. 271, 16784-16791. 


\section{FIGURE LEGENDS}

Figure 1. In vitro DNA topoisomerase I cleavage site on TTA sequence. 1,5 and $10 \mathrm{U}$ of calf thymus DNA topoisomerase I (triangle) were reacted with the 3' labelled TTA containing DNA sequence in the presence of Camptothecin (CPT). Mapping of the fragments, separated by sequencing gel, was obtained comparing the migration of samples with the size marker (M) and sequencing lane of $\mathrm{A}$ (A). Black arrows and asterisks indicate in vitro DNA topoisomerase I cleavages on TTA or different sequences respectively. A schematic map containing the genes flanking the TTA repeated sequence is reported at the right side. Numbering refers to PRE8 ATG.

\section{Figure 2. In vivo DNA topoisomerase I cleavage site on TTA sequence}

Spheroplasts from WT or top1 $\triangle$ cells were treated with 0 (U), 50, 100 and $200 \mu \mathrm{M}$ CPT (triangles). The fragments resulting from DNA topoisomerase I activity were primer extended from oligonucleotide VEC (white arrow) annealing at position -499 from PRE8 ATG. Black arrows indicate DNA topoisomerase I cleavage sites. M: pBR322/MspI, size marker. A: sequencing lane. At the right part of the figure a representation of the analyzed region is shown.

Figure 3. Nucleosome positioning on the TTA sequence

Panel A. Nystatin-permeabilized spheroplasts were treated with 0,2 , and $4 \mathrm{U}$ respectively of micrococcal nuclease (MNase). Purified DNA was restricted with BamHI and run in 1.2\% agarose gel, transferred onto a nitrocellulose membrane and hybridised with the TTA probe. Chromatin: in vivo digested samples; Naked: in vitro digested samples of deproteinized DNA; U: untreated DNA; M1: size marker (1kb ladder); M2: size marker (123bp ladder). Black arrows indicate MNase cleavage sites.

Panel B: DNA was digested in vivo (Chromatin) and in vitro (Naked DNA), after deproteinization, with 0-1-2-4-8 U of MNase and primer extended with the labelled oligonucleotide VEC (position 449). Lane $\mathrm{M}$ size marker (pBR322/MspI); A: sequencing lane; U: untreated DNA. Filled and empty arrowheads indicate protections and enhancements respectively; map on the right shows nucleosome with multiple positions (ellipses). All numbering refers to PRE8 ATG.

Figure 4. Nucleosome organization on TTA sequence upon nucleosomes removal.

Panel A: Spheroplasts from cells grown in galactose (GAL) (transcribing H4 gene) or in glucose (GLU) (non-transcribing H4 gene) were subjected to MNase digestion (as in Figure 3). DNA was 
purified and analyzed by agarose gel electrophoresis. Lane M: size marker (1kb ladder); U: untreated DNA. Arrows show cleavages of MNase on DNA.

Panel B: Spheroplasts from cells grown in galactose (GAL) (transcribing $\mathrm{H} 4$ gene) or in glucose (GLU) (non-transcribing $\mathrm{H} 4$ gene) were subjected to MNase digestion as reported in Figure 3 and compared with MNase digestions on deproteinized DNA (Naked). Lane M: size marker (1kb ladder); U: untreated DNA; ellipses indicate nucleosomes.

Figure 5. DNA topoisomerase I cleavage activity on TTA sequence upon nucleosomes removal Panel A: Spheroplasts from cells grown in galactose (GAL) (transcribing $\mathrm{H} 4$ gene) or in glucose (GLU) (non-transcribing H4 gene) were subjected to DNA topoisomerase I cleavage sites in the presence of camptothecin; induction as in Figure 2. Lane M: size marker (pBR322/MspI); U: DNA from untreated cells. Arrows indicate DNA topoisomerase I cleavage sites. Numbering refers to PRE 8 ATG. At the right part of the figure a schematic representation of the analyzed region is shown.

Panel B: Samples as in Panel A were primer extended from oligonucleotide r3 in order to show DNA topoisomerase I cleavage sites on NTS2 region of ribosomal genes. C, T: sequencing lanes. U: untreated sample; M: size marker.

Figure 6. DNA topoisomerase I accessibility on different contexts. Different DNA topoisomerase I digestion profiles from: in vitro (white bar), in vivo (dashed bar), in vivo GLU (non-transcribing H4 gene) (black bar) and in vivo GAL (transcribing H4 gene) (dotted bar) where evaluated by densitometric scanning. Data represent the \% of cleavage activity on the TTA repeat. 
B) Biochemical Journal Immediate Publication. Published on 30 Oct 2007 as manuscript BJ20070893

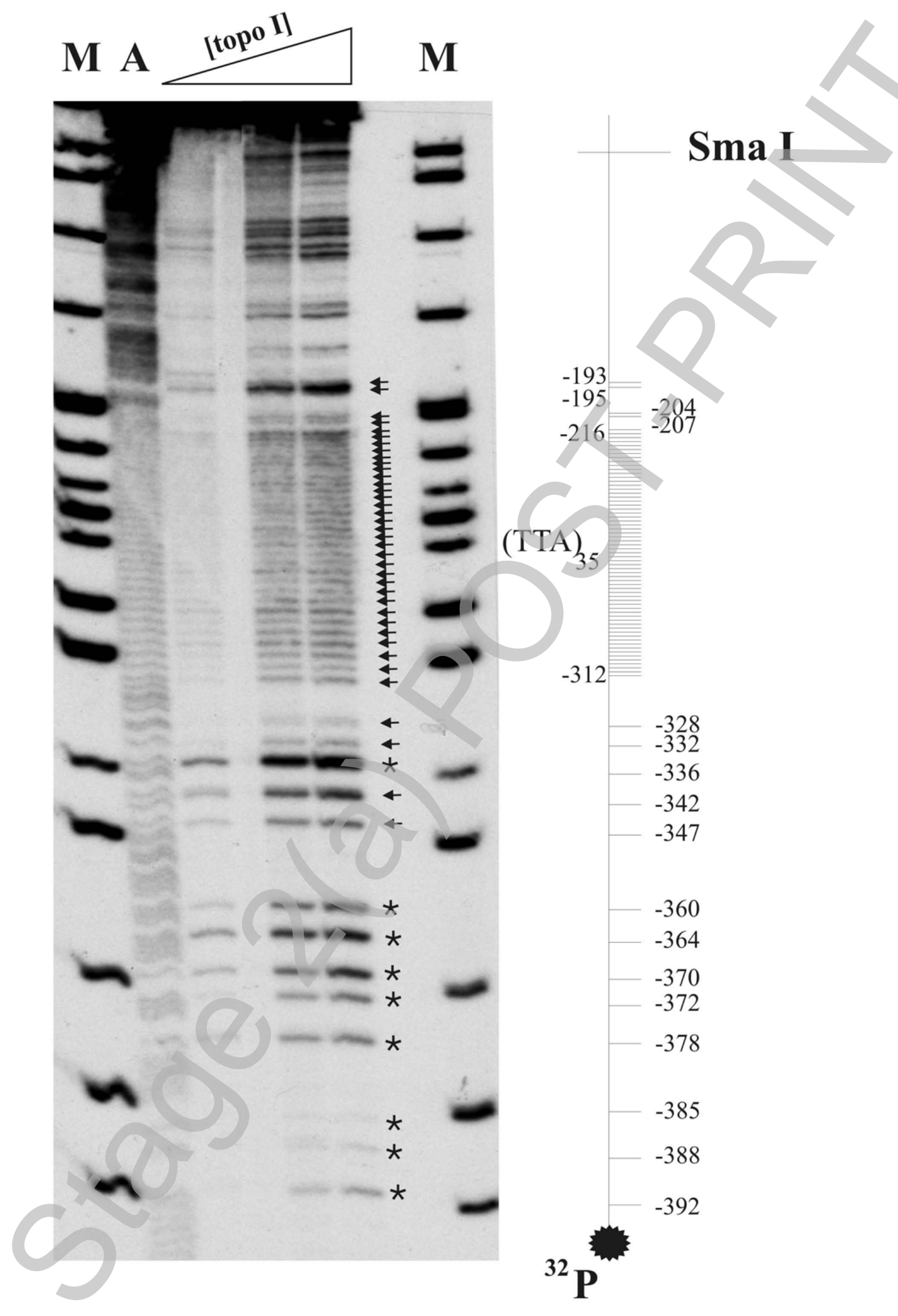


WT
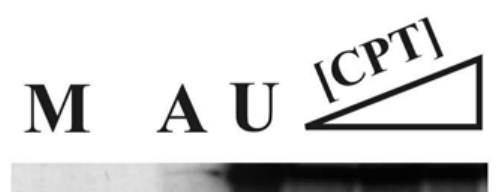

-

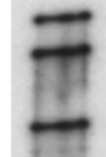

(1)
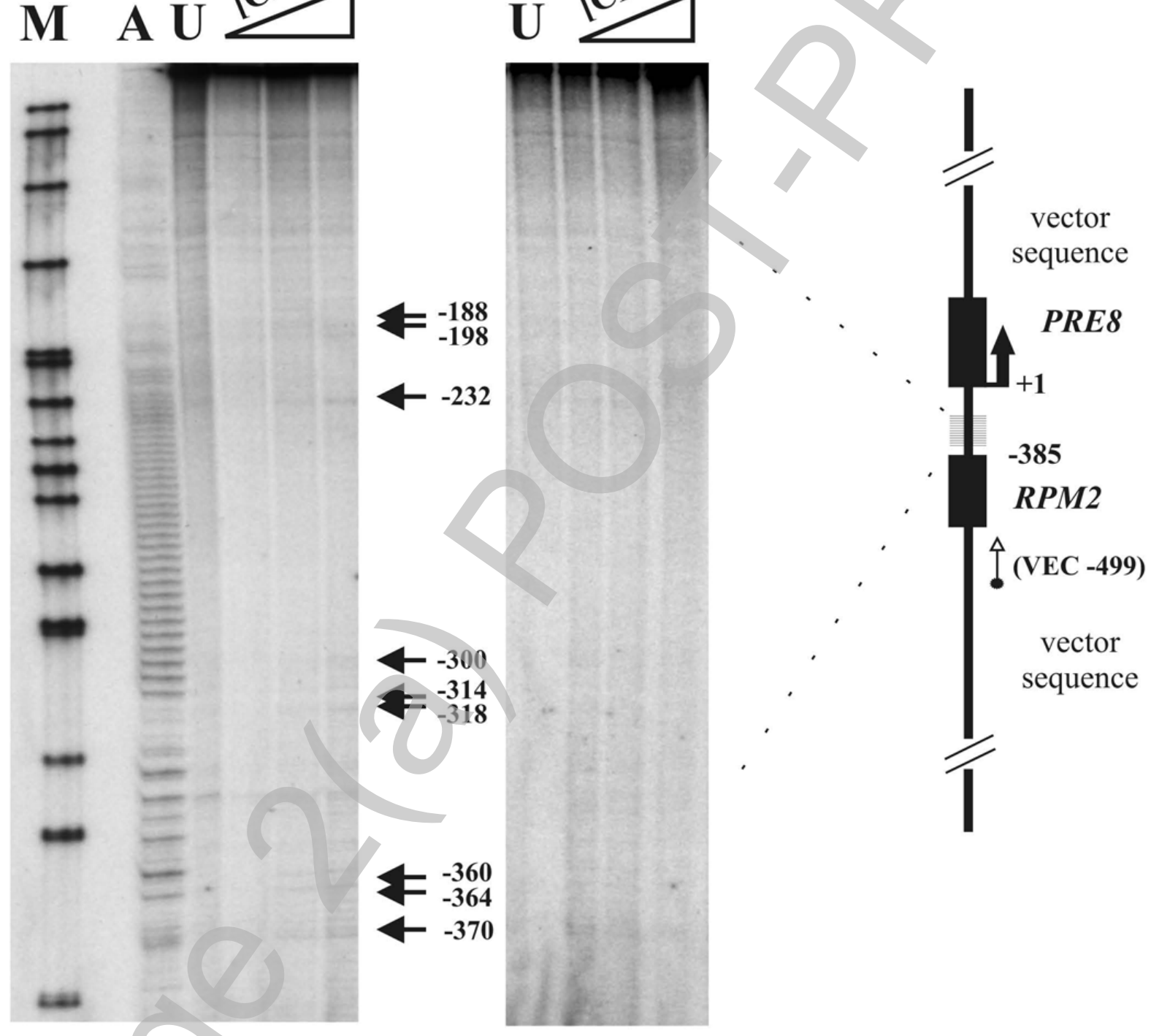
A

B

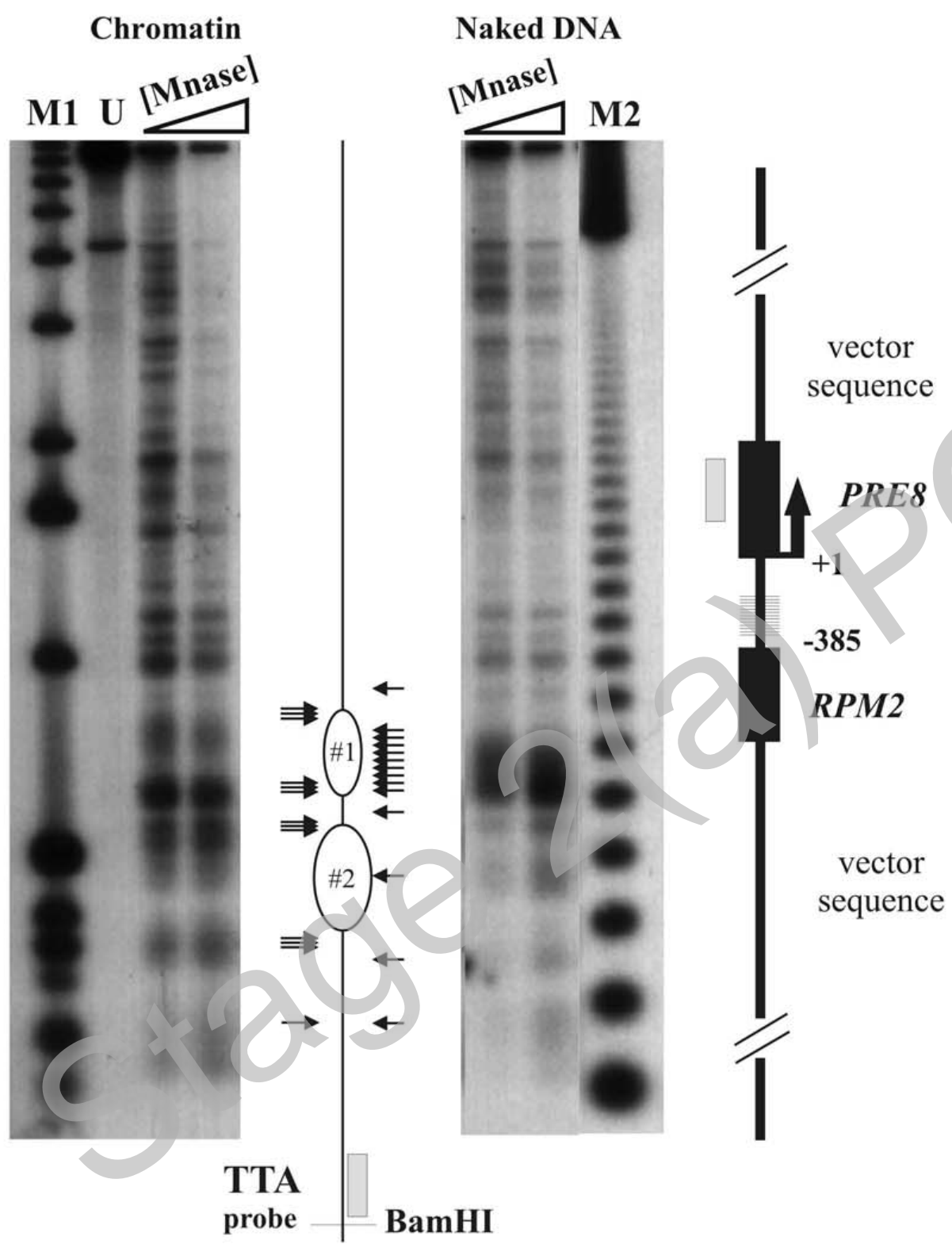

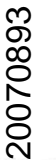

Chromatin Paked DNA

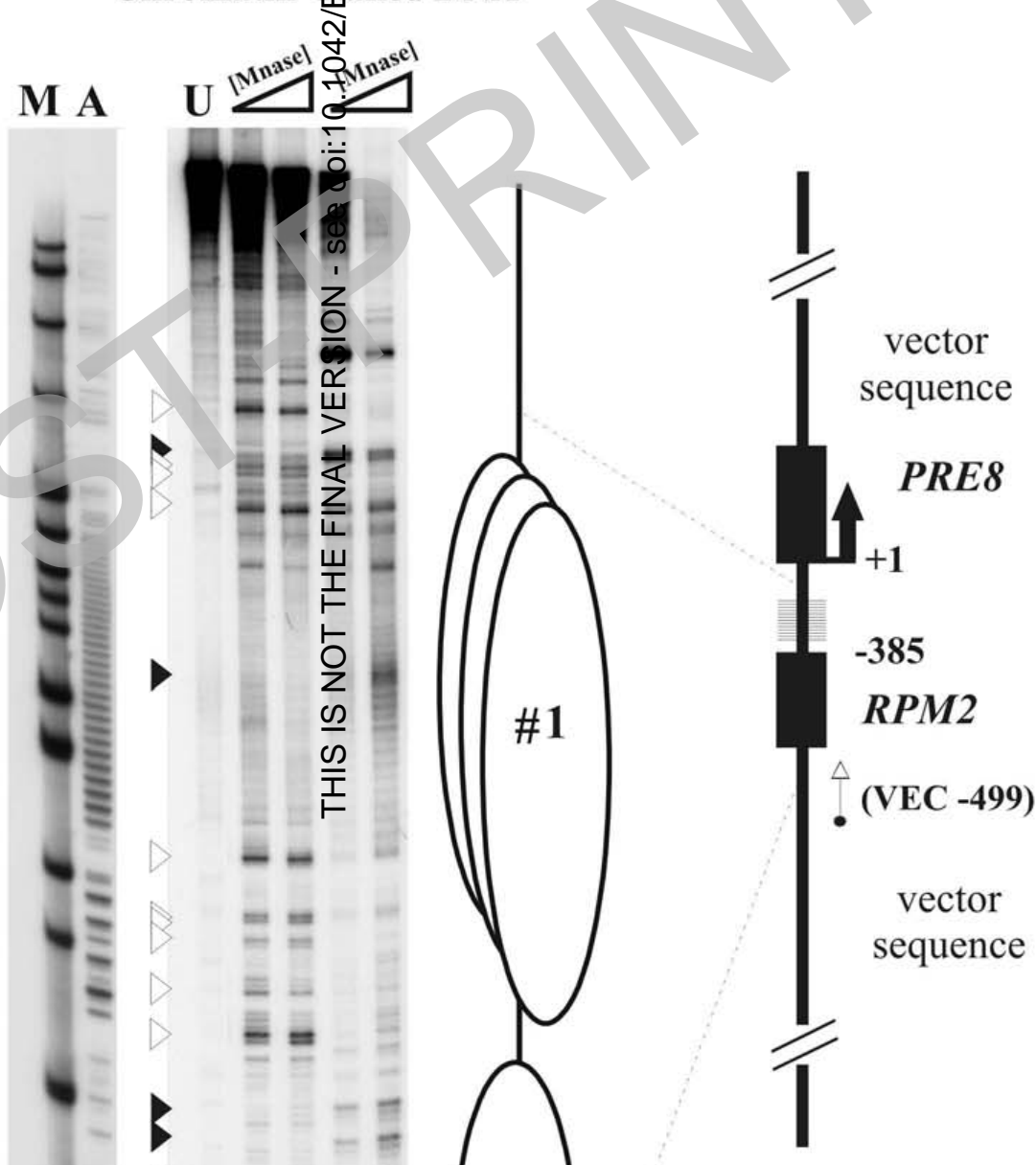

Licenced copy. Copying is not permitted, except with prior permission and as allowed by law. @ 2007 The Authors Journal compilation @ 2007 Biochemical Society 


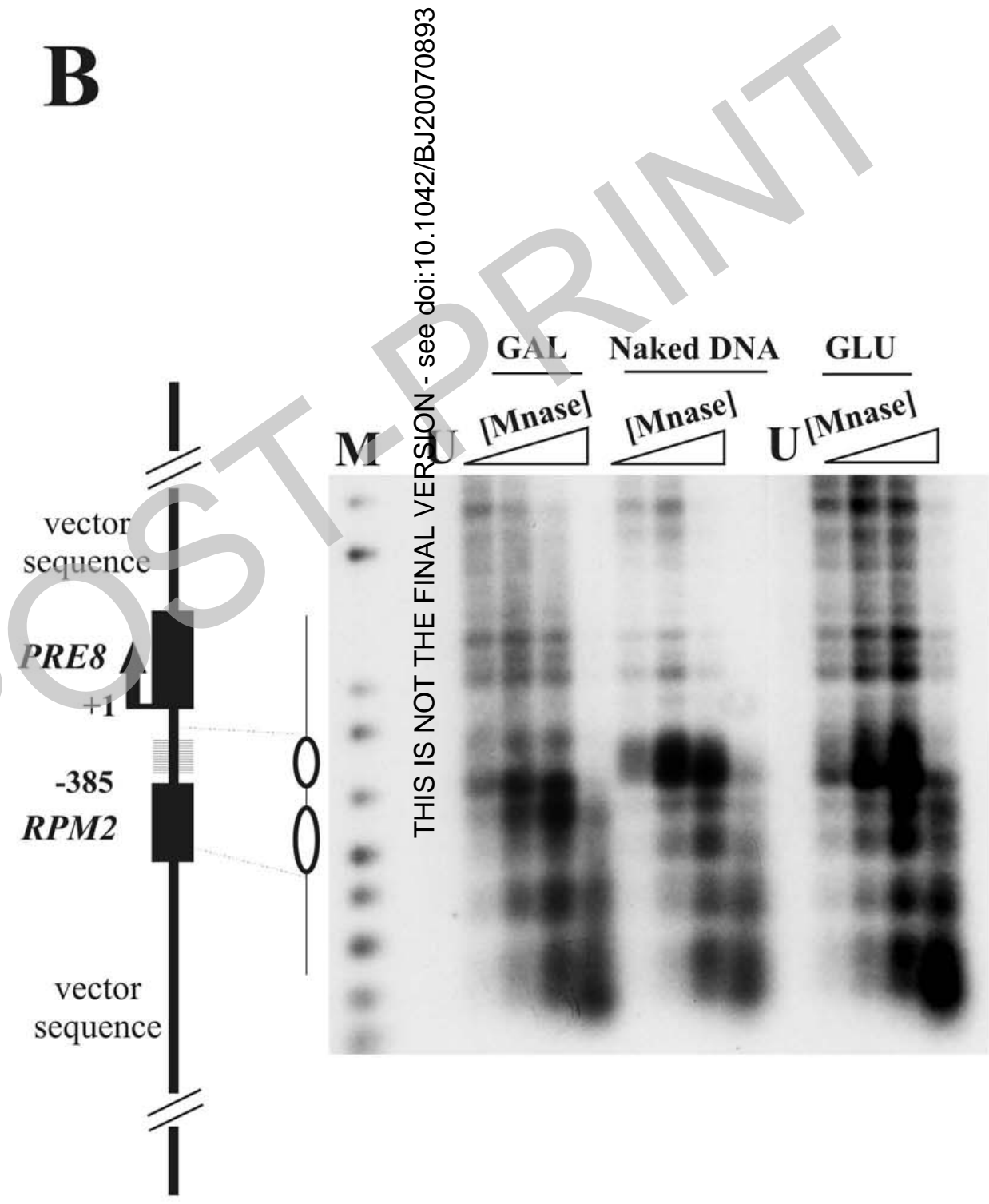




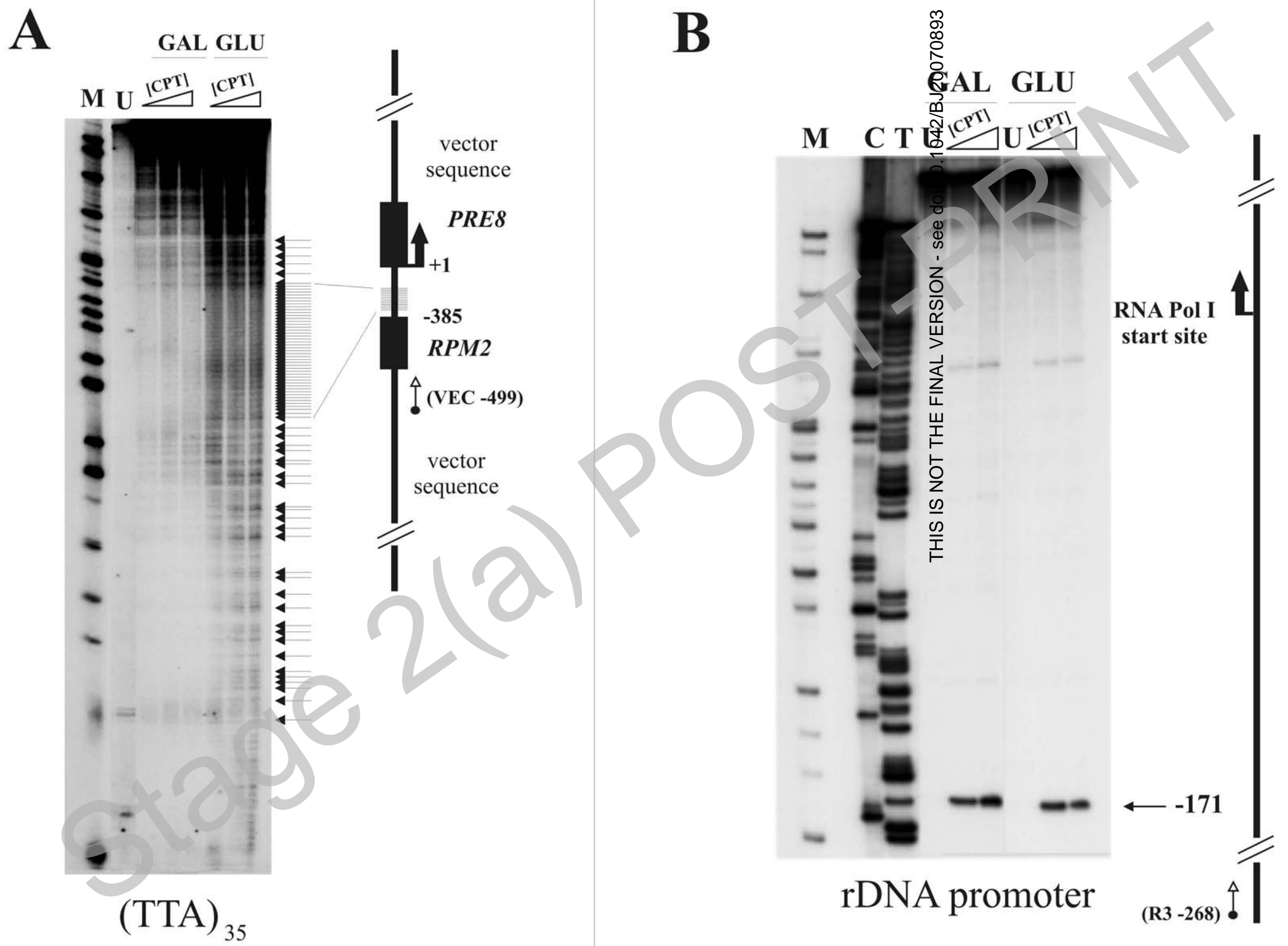

Licenced copy. Copying is not permitted, except with prior permission and as allowed by law. (c) 2007 The Authors Journal compilation (@ 2007 Biochemical Society 


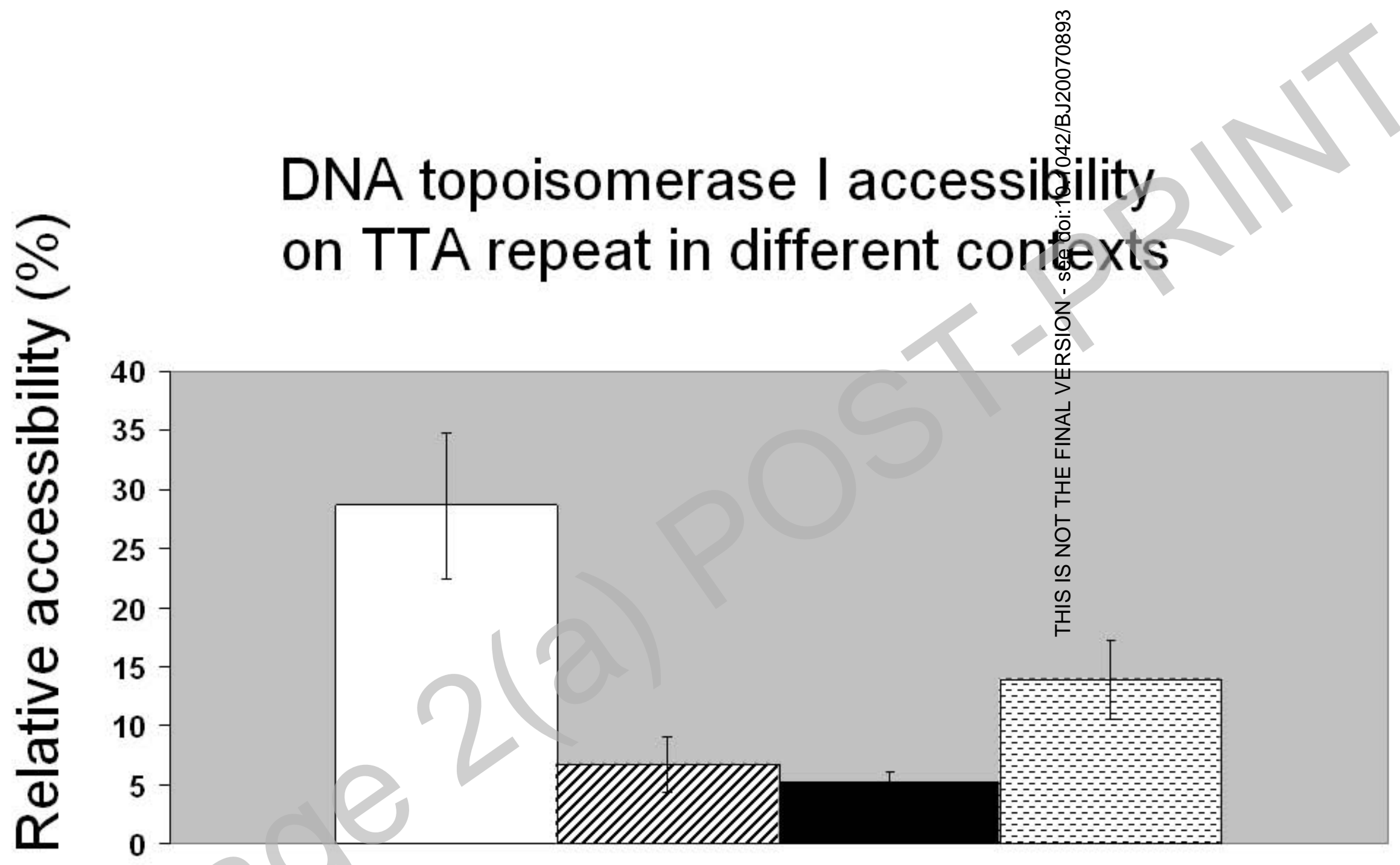

